\title{
Middle East Pain Syndrome (MEPS) Vitamin D3 Deficiency Induced Hyperparathyroidism And Fibromyalgia Mimicking Rheumatoid Arthritis
}

Adel Elbeialy ( $\sim$ aelbeialy@azhar.edu.eg )

Al-Azhar University Faculty of Medicine for Girls https://orcid.org/0000-0002-5034-8545

Abdlnby Bauomi

Al-Azhar University Faculty of Medicine

Basma Elnaggar

Al-Azhar University Faculty of Medicinefor Girls

Hala Elzomor

Al-Azhar University Faculty of Medicinefor Girls

\section{Article}

Keywords: SHPT, RA, VitaminD3, Cadmium, Fibromyalgia

Posted Date: August 24th, 2021

DOl: https://doi.org/10.21203/rs.3.rs-820285/v1

License: (c) (i) This work is licensed under a Creative Commons Attribution 4.0 International License.

Read Full License 


\section{Abstract}

Objectives: Musculoskeletal pains are sometimes misdiagnosed in some diseases, like rheumatoid and psoriatic arthritis, erosive OA, etc. Secondary hyperparathyroidism was not considered a differential diagnosis for RA, despite the fact that it can cause arthralgia or arthritis. Also, fibromyalgia is a psychosomatic condition marked by widespread pain and tenderness.

Methods: This study included 400 patients attended outpatient clinics of Al-Azhar Faculty of medicine in Egypt, and Elaj Specialized Clinics in Saudi Arabia, who were not fulfilling criteria for RA diagnosis, and not responding to its treatment. Other diseases like psoriatic arthritis and erosive OA were excluded. Criteria for classification of fibromyalgia syndrome were applied to all patients. We did lab tests of RF, ACPA, ESR, CRP, LFT, RFT, vitamin D3, PTH, calcium, phosphorus, and SUA. Radiological imaging modalities for diagnosis or exclusion of suspected diseases were applied.

Results: All patients were fulfilling both old and new criteria of fibromyalgia syndrome, and not fulfilling any RA criteria, $18 \%$ were seropositive with low RF titers and negative ACPA. All patients had vitamin D3 deficiency or insufficiency. $75 \%$ of patients had abnormally high levels of PTH, and had no parathyroid gland pathology. X-rays showed subperiosteal and subchondral resorption of mainly thumbs, subchondral osteopenia of proximal and middle phalanges, mild subperiosteal resorption along the radial aspect of the middle phalanx and mild tuft erosions, besides changes in the carpus closely resembling those of rheumatoid arthritis, of ulnar styloid resorption, radiocarpal and scapho-trapezoid joint arthritis. Of special interest, the presence of tuft spurs-like excrescences.

\section{Introduction}

Secondary hyperparathyroidism (SHPT) is a medical disorder in which the parathyroid glands secrete too much parathyroid hormone (PTH) in reaction to hypocalcemia, resulting in gland hyperplasia. The most prevalent cause of SHPT is chronic renal failure, which is caused by the kidneys' inability to convert enough vitamin D2 to its active form D3, and hence to not adequately excrete phosphate. Insoluble calcium phosphate occurs in the body as a result, removing calcium from circulation. Both of these mechanisms result in hypocalcemia and, as a result, SHPT [1]. Malabsorption (chronic pancreatitis, small bowel disease, malabsorption-dependent bariatric surgery) can also cause SHPT because fat-soluble vitamin $\mathrm{D}$ cannot be reabsorbed. As a consequence, hypocalcemia develops, and parathyroid hormone secretion rises in an attempt to raise serum calcium levels. Other factors include a calcium shortage in the diet, a vitamin D deficiency, or steatorrhea. [ 2]

Muscle cramps and bone pain are common symptoms of HPT, as are perioral tingling or paresthesia in the fingers or toes, and positive Chvostek and Trousseau signs. Only a few cases of arthritis mimicking rheumatoid arthritis as a presenting characteristic of HPT have been described in the literature. [3]

Rheumatoid arthritis (RA) is a chronic autoimmune disease that affects the joints principally which become warm, swollen, and painful. Wrists and hands are the most commonly affected joints, with the 
same joints being impacted bilaterally. It was previously established that RF is not exclusive to RA and can be present in other disorders such as Epstein-Barr virus or Parvovirus infections, as well as in 5 to $10 \%$ of the normal population, especially elderly people. $[4,5]$.

Anti-CCP antibodies are produced locally at areas of inflammation, not just in the synovium of RA patients, but also in non-RA conditions. As a result, anti-CCP positivity may be seen in a subset of patients with diseases other than RA. [6]

One of the reasons of SHPT, as we know, is vitamin D3 deficiency, which has been discovered to be frequent in the Middle East, despite the region's abundance of sun rays and hence UV exposure. Heavy metal pollution, such as cadmium and lead, is one of the most likely causes of vitamin D insufficiency in the Middle East. [7,8]

The mitochondria of the kidney's proximal convoluted tubule cells produce circulating 1a,25dihydroxyvitamin D3 [1,25(OH)2D3], and parathyroid hormone (PTH) evidently increases 1,25(OH)2D3 production while 1,25(OH)2D3 suppresses PTH production. [9]

Cadmium prefers to accumulate in the kidneys. The accumulation of cadmium-metallothionein (CdMT) in the proximal convoluted tubules causes damage as a consequence to renal cadmium deposition.

The preferential absorption of CdMT into the $\mathrm{S} 1$ and $\mathrm{S} 2$ segments of the proximal tubules, which is responsible for converting 25(OH)D3 to 1,25(OH)2D3, may contribute to CdMT-induced nephrotoxicity. [10].

Vitamin D3 deficiency and hyperparathyroidism have been linked to Fibromyalgia syndrome $[11,12]$. Meanwhile, gout, pseudogout, juxtaarticular erosions, tenosynovitis, periarthritis, and pericapsular calcification are just a few of the articular symptoms linked to hyperparathyroidism [13].

\section{Patients And Methods}

This study included 400 patients attended outpatient clinics of Al-Azhar University Faculty of Medicine Hospitals, Egypt, and Elaj Specialized Clinics, Saudi Arabia, in the period between Nov 2014 to Dec 2019, who were previously diagnosed as RA, but we found them not fulfilling any criteria for RA diagnosis, and not responding to treatments including NSAIDs, and DMARDs, (Table 1).

\section{Inclusion and exclusion criteria:}

We got consent from all patients to re-evaluate and re-diagnose them. They were investigated to establish existence of RA, using criteria for classification of RA [14, 15], with exclusion of other mimicking diseases like psoriatic arthritis, erosive $O A$, viral arthritis, reactive arthritis, IBD arthritis, Lyme's disease, and palindromic rheumatism. In addition, we applied old and new criteria for classification of fibromyalgia syndrome to all patients $[16,17]$ to establish the diagnosis of FMS. 
We did lab tests of RF, ACPA, ESR, CRP, LFT, RFT, serum 25 cholcalciferol, PTH, total and ionized calcium, phosphorus, and SUA. In addition, all patients did plain X-ray (Toshiba Digital Radiography System, DIGIX U) for hands, knees, and lumbar regions. When recommended, they were exposed to MRI (Philips1.5 tesla), and CT (Multi-Slice spiral CT Aston). High-resolution peripheral quantitative computed tomography (HR-pQCT) imaging allowed for highly reliable assessment of erosion in patients suspected to have RA to exclude them.

\section{Results}

All patients scheduled were fulfilling both old and new criteria of fibromyalgia syndrome, and not fulfilling any RA criteria, $82 \%$ of them were seronegative and $18 \%$ were seropositive with low RF titers and negative ACPA. All patients had vitamin D3 deficiency or insufficiency. $75 \%$ of patients had abnormally high levels of PTH $(96 \pm 12 \mathrm{pg} / \mathrm{ml})$, and had no parathyroid gland pathology; hence we called them SHPT group. (Tables 2, 3)

X-rays of our patients hands showed subperiosteal and subchondral resorption of mainly thumbs, subchondral osteopenia of proximal and middle phalanges, mild subperiosteal resorption along the radial aspect of the middle phalanx (88\%) and mild tuft erosions (12\%), besides changes in the carpus closely resembling those of rheumatoid arthritis, of ulnar styloid resorption, radiocarpal and scapho-trapezoid joint arthritis (35\%). Of special interest, the presence of tuft spurs-like excrescences (95\%) mimicking that of (spade phalanx sign) of acromegaly, but without any other criteria of acromegaly (Fig.1, 2, 3). Plain X ray of knees showed chondrocalcinosis (20\%), intracortical resorption, and osteopenia (Fig 4). Lumbar spine shows mild sclerotic bands of the Superior and inferior end plates mimicking rugger jersey sign (30\%), as well as osteopenia and brown tumors (30\%).we also detected calcaneal spurs in $45 \%$ of patients. (table 4).

\section{Discussion}

We found many patients with bilateral wrist and hand arthritis had a combination of vitamin D3 deficiency, increased serum parathyroid hormone, and fibromyalgia. They were misdiagnosed as having either seronegative or seropositive rheumatoid arthritis, resulting in treatment failure and long-term pain. We revaluated them as having a new syndrome, of vitaminD3 deficiency, FMS, and SHPT, we named it Middle East Pain Syndrom (MEPS), since all patients we discovered were from Middle East.

A study by Helliwell showed arthritis in 11\% of HPT patients, including two cases of erosive arthritis [18]. Also, HPT presenting as arthritis had been described in other case reports [18-22]. Meanwhile, HPT itself, can occasionally co-exist with inflammatory arthritis like rheumatoid arthritis [23]. In addition to clinical and biochemical features, radiographical features were helpful to differentiate both. HPT does not cause only osteoporosis and pathological fractures, it can cause also bone erosions, which are shaggy in appearance and distributed in the radiocarpal, radioulnar, metacarpophalangeal and distal interphalangeal joints, in contrast to that which predominate PIP joints of rheumatoid arthritis [24]. 
HPT is characterized by both bone resorption and formation. Plain radiography results may include resorption and sclerosis of multiple places in the skeletal system as a result [25]. Bone findings were characterized by the osteoclastic resorption of bone, osteoblastic bone formation, and fibrous replacement of marrow, with radiographic findings of subperiosteal resorption, brown tumors, bone cysts, and sclerosis. [26]

The disease may progress with further destruction that results in a ground-glass appearance in bone trabeculae [26]. Bone resorption may be interpreted as subperiosteal, intracortical, trabecular, endosteal, subchondral, subligamentous, or subtendinous. Subperiosteal bone resorption is an early and virtually pathognomonic sign of HPT, which is marked by marginal erosions with adjacent resorption of bone and sclerosis. Although subperiosteal bone resorption can affect many sites, the most common site in HPT is the middle phalanges of the index and middle fingers, primarily on the radial aspect [26]. HPT osteoporosis attains a characteristic tubal, cigar or pyramidal like appearance, other than that found in RA or other collagen diseases. Of special interest, the presence of tuft spur-like excrescences mimicking that of spade phalanx sign of acromegaly [27], but without any other criteria of acromegaly. One literature reported this sign in an unexplained case report, [28].

Of course, the secondary hyperparathyroidism found in our patients is a sequence of chronic vitamin D3 deficiency or insufficiency, and not associated with renal problems, nor malignancies, or other precipitating factors. We attributed this chronic vitamin D3 deficiency or insufficiency to probable long term exposure to pollutants, as the heavy metals cadmium and lead, present in some mineral waters, fizzy waters, fried snacks, and tobacco smoke. [7, 8, 29].

Vitamin $D$ is produced in the skin from 7-dehydrocholesterol by UV irradiation. The liver and other tissues metabolize vitamin $D$, whether from the skin or oral ingestion, to $250 \mathrm{HD}$, the principal circulating form of vitamin $\mathrm{D}$. $250 \mathrm{HD}$ is then further metabolized to 1,25(OH)2D principally in the kidney, by the enzyme CYP27B1. 1,25(OH)2D is the principal hormonal form of vitamin $D$, responsible for most of its biologic actions. [30]

Cadmium preferentially selects to settle in kidneys. Renal cadmium deposition induces injury resulted from accumulation of cadmium-metallothionein (CdMT) in the proximal convoluted tubules. CdMTinduced nephrotoxicity might be due, at least in part, to its preferential uptake of CdMT into the S1 and S2 segments of the proximal tubules, which is responsible for converting 25(OH)D3 to 1,25(OH)2D3.[31].

The mitochondria of proximal convoluted tubule cells of the kidney are the site of production of circulating 1a,25-dihydroxyvitamin D3 . Parathyroid hormone (PTH) markedly stimulates 1,25(OH)2D3 production, while 1,25(OH)2D3 suppresses production of PTH itself.[9]

Vitamin D3 deficiency and HPT are established to be associated with Fibromyalgia syndrome [32,33]. Hyperparathyroidism is known to be associated with multiple articular manifestations including gout, pseudogout, juxtaarticular erosions, tenosynovitis, periarthritis, and pericapsular calcification [12]. Hyperparathyroidism is a cause of bone pain and aching joints due to osteoporosis or osteopenia 
because of the continued removal of calcium from bone as well as chondrocalcinosis and pseudogout. Bone pain may also be caused by hemorrhages within the bone. Joint pains, degenerative arthritis, joint laxity and muscle weakness can result from calcium pyrophosphate deposition. [34]. This chronic suffering from vitamin D3 deficiency or insufficiency induces SHPT, which in turn induces musculoskeletal perturbance in the form of muscle and tendon pain and weakness, bone and joint pains.

This syndrome responds well to treatment of fibromyalgia regimen with SSRI, muscle relaxants, and vitamin D3 supplementation 5000-10000 iu/day, antioxidants including selenium, vitamins E and C. In cases with arthritis we add sulphasalazine $1 \mathrm{gm} /$ bid or Plaquenil $200 \mathrm{mg} / \mathrm{bid}$ to which their inflamed joints respond significantly.

\section{Conclusion}

Middl East Pain Syndrome is a new disease, which must be given more attention for diagnosis, and more extensive studies for its pathogenesis, prevention. and cure. Cadmium pollution must be considered as one of the new millennium big challenges that should be treated efficiently, to prevent many economically affecting health hazards.

\section{Declarations}

All authors confirm that there are no any potential conflicts of interest of each of them. We confirm hereby that the manuscript has not been submitted or is not simultaneously being submitted elsewhere, is not at the time of submission under consideration by another journal or other publication, and that no portion of the data has been or will be published elsewhere while the manuscript is under review by the journal, unless rejected or withdrawn by the author. Also we confirm that no portion of the data has been or will be published elsewhere while the manuscript is under review by the journal.

We confirm also that there is not any financial support or other benefits from commercial sources for the work reported on in the manuscript, or any other financial interests that any of the authors may have, which could create a potential conflict of interest or the appearance of a conflict of interest with regard to the work.

Ethics: This study was carried out according to regulations and approval of Ethics Committee of Faculty of Medicine for Girls, Al-Azhar University, Nasr City, Cairo, Egypt, Registered at Central Administration of Research \& Development; Egyptian Ministry of Health: Reg No. RHBIRB2018122001

\section{References}

1. TaylorJG, BushinskyDA. Calcium and Phosphorus Homeostasis. Blood Purif 2009;27:387-394.

2. PepeJ, CiprianiC, PilottoR, De LuciaF, CastroC, LengeL, et al. Sporadic and hereditary primary hyperparathyroidism. J Endocrinol Invest. 2011; 34: 40-44. 
3. MadkhaliT, AlhefdhiA, ChenH, ElfenbeinD. Primary hyperparathyroidism. Ulus Cerrahi Derg. 2016;32:58-66.

4. FalkenburgWJJ. IgG Subclass Specificity Discriminates Restricted IgM Rheumatoid Factor Responses From More Mature Anti-Citrullinated Protein Antibody-Associated or Isotype-Switched IgA Responses. Arthritis \& Rheumatology. 2015; 67: 3124-3134.

5. EdkinsA, CushleyW. The Jekyll and Hyde nature of antibodies. Biological Sciences Review. 2012; 25: 2- 4 .

6. VossenaarER, SmeetsTJ, KraanMC, RaatsJM, van VenrooijWJ, TakPP. The presence of citrullinated proteins is not specific for rheumatoid synovial tissue. Arthritis Rheum. 2004; 50: 3485-94.

7. ElbeialyA, Hesham Eldesouky. AB1261 Cadmium toxicity as a probable cause of osteopenia in adolescents and its relation to bad dietary habits. 2019: 2091-2092.

8. KazantzisG. Cadmium, osteoporosis and calcium metabolism. Biometals. 2004; 17: 493-498.

9. WangY, ZhuJ, DeLucaHF. The vitamin D receptor in the proximal renal tubule is a key regulator of serum 1a,25-dihydroxyvitamin D囚. Am J Physiol Endocrinol Metab. 2015; 308:E201-5.

10. KurataY, KatsutaO, DoiT, et al. Chronic Cadmium Treatment Induces Tubular Nephropathy and Osteomalacic Osteopenia in Ovariectomized Cynomolgus Monkeys. Veterinary Pathology. 2014; 51: 919-931.

11. CostaJM, RanzolinA, Costa NetoCA, MarquesCD, DuarteAL. High frequency of asymptomatic hyperparathyroidism in patients with fibromyalgia: random association or misdiagnosis?. Revista brasileira de reumatologia. 2016; 56: 391-7.

12. BorgiaAR, CavallascaJA, CostaCA, MusuruanaJL. Hyperparathyroidism, a forgotten cause of musculoskeletal pain. Reumatol Clin. 2012 ; 8: 299-301.

13. Mackenzie-FederJ, SirrsS, AndersonD, SharifJ, KhanA. Primary hyperparathyroidism: an overview. Int J Endocrinol. 2011; Article.

14. ArnettFC, EdworthySM, BlochDA, McShaneDJ, FriesJF, CooperNS, et al. The American Rheumatism Association 1987 revised criteria for the classification of rheumatoid arthritis. Arthritis Rheum. 1988; 31: 315-24.

15. AletahaD, NeogiT, SilmanAJ, FunovitsJ, FelsonDT, BinghamCO, et al. 2010 Rheumatoid arthritis classification criteria: an American College of Rheumatology/European League Against Rheumatism collaborative initiative. Arthritis Rheum. 2010; 62:2569-81

16. WolfeF, SmytheHA, YunusMB, BennettRM, BombardierC, GoldenbergDL, et al. The American College of Rheumatology 1990 Criteria for the Classification of Fibromyalgia. Report of the Multicenter Criteria Committee. Arthritis Rheum. 1990; 33:160-72.

17. WolfeF, ClauwDJ, FitzcharlesMA, GoldenbergDL, KatzRS, MeaseP, et al. The American College of Rheumatology preliminary diagnostic criteria for fibromyalgia and measurement of symptom severity. Arthritis Care Res. 2010; 62:600-10. 
18. HelliwellM. Rheumatic symptoms in primary hyperparathyroidism. Postgrad Med J. 1983;59: 236240.

19. BywatersEGL, Dixon ASJ, ScottJT. Joint lesions of hyperparathyroidism. Annals of the Rheumatic Diseases. 1963; 22: 171.

20. ZvaiflerNJ, ReefeWE, BlackRL. Articular manifestations in primary hyperparathyroidism. Arthritis Rheum.1962; 5: 237-249

21. LipsonRL, WilliamsLE, ZvaiflerNJ, HowellDS. The "connective tissue disorder" of hyperparathyroidism. Arthritis \& Rheumatism: Official Journal of the American College of Rheumatology. 1968;11: 198-205.

22. DuraywishAA. Chronic unclassified polyarthritis: A rare presentation of primary hyperparathyroidism. Med J Cairo Univ. 2016; 84:265-269.

23. SalariM, AboutorabiRB, RezaieyazdiZ. Hypercalcemia and Lytic Bone Lesions Masquerading Inflammatory Arthritis Treated as Rheumatoid Arthritis. Arch Iran Med. 2015;18: 720-3.

24. FengX, McDonaldJM. Disorders of bone remodeling. Annu Rev Pathol. 2011; 6: 121-145.

25. LiN, LiXM, XuL, SunWJ, ChengXG, TianW. Comparison of QCT and DXA: osteoporosis detection rates in postmenopausal women. International Journal of Endocrinology. 2013.

26. PatelAA, RamanathanR, KubanJ, WillisMH. Imaging findings and evaluation of metabolic bone disease. Advances in Radiology. 2015.

27. KillingerZ, PayerJ, Lazúrovál, ImrichR, HomérováZ, KužmaM, Rovenský J. Arthropathy in acromegaly. Rheum Dis Clin North Am. 2010; 36: 713-20.

28. FreyschmidtJ, BrossmannJ, SternbergA et al. Freyschmidt's "Koehler/Zimmer's Borderlands of Normal and Early Pathological Findings in Skeletal Radiography". 5 rev. 2003.

29. Elbeialy, A. A., and H. A. Eldosouky. "AB0984 Cadmium toxicity as a probable cause of smoking induced bone loss." (2018): 1614-1614.

30. Bikle D. Vitamin D: Production, Metabolism, and Mechanisms of Action. [Updated 2017 Aug 11]. In: Feingold KR, Anawalt B, Boyce A, et al., editors. Endotext [Internet]. South Dartmouth (MA): MDText.com, Inc.; 2000-. Available from: https://www.ncbi.nlm.nih.gov/books/NBK278935/

31. Johri N, Jacquillet G, Unwin R. Heavy metal poisoning: the effects of cadmium on the kidney. Biometals. 2010 Oct;23(5):783-92. doi: 10.1007/s10534-010-9328-y. Epub 2010 Mar 31. PMID: 20354761.

32. Okumus M, Koybası M, Tuncay F, Ceceli E, Ayhan F, Yorgancioglu R, Borman P. Fibromyalgia syndrome: is it related to vitamin D deficiency in premenopausal female patients? Pain Manag Nurs. 2013 Dec;14(4):e156-e163. doi: 10.1016/j.pmn.2011.09.006. Epub 2011 Dec 20. PMID: 24315268.

33. Bellato E, Marini E, Castoldi F, et al. Fibromyalgia syndrome: etiology, pathogenesis, diagnosis, and treatment [published correction appears in Pain Res Treat. 2013;2013:960270]. Pain Res Treat. 2012;2012:426130. doi:10.1155/2012/426130 
34. Jeremy G. Jones, CHAPTER 32 - Biochemistry of articular disorders, Editor(s): William J. Marshall, Marta Lapsley, Andrew P. Day, Ruth M. Ayling, Clinical Biochemistry: Metabolic and Clinical Aspects (Third Edition), Churchill Livingstone, 2014, Pages 636-645, ISBN 9780702051401,

\section{Tables}

Table 1: Demographic data of MEPS study group.

\begin{tabular}{|lllllll|}
\hline & No & $\%$ & Age & \multicolumn{2}{c|}{ Female Sex } & Duration \\
\cline { 7 - 8 } MEPS & & & years & N & $\%$ & years \\
\hline Seropositive & 72 & $18 \%$ & $40 \pm 13$ & 220 & $55 \%$ & $1.27 \pm 0.82$ \\
\hline Seronegative & 328 & $82 \%$ & $39 \pm 16$ & 180 & $45 \%$ & $1.17 \pm 0.90$ \\
\hline Total & 400 & $100 \%$ & & & & $1.22 \pm 0.86$ \\
\hline
\end{tabular}

Table 2: Laboratory chemistry and PTH results of MEPS study group

\begin{tabular}{|lllllllll|}
\hline & No & ESR & SUA & Ca & Ca++ & D3 & PTH & P \\
& & $\mathrm{mm}$ & $\mathrm{mg} / \mathrm{dl}$ & $\mathrm{mg} / \mathrm{dl}$ & $\mathrm{mg} / \mathrm{dl}$ & $\mathrm{ng} / \mathrm{ml}$ & $\mathrm{pg} / \mathrm{ml}$ & $\mathrm{mg} / \mathrm{dl}$ \\
\hline MEPS & 400 & 40 & 6.4 & 8.6 & 1.11 & 15.58 & 96 & 4.8 \\
& & \pm 14 & \pm 1.3 & \pm 1.4 & \pm 0.11 & \pm 6.66 & \pm 12 & \pm 1.2 \\
\hline
\end{tabular}

ESR:erythrocyte sedimentation rate, SUA: serum uric acid, Ca: total calcium, Ca++: ionized calcium, D3: vitamin D3, PTH: parathyroid hormone, P: serum phosphorus

Table 3: Different Criteria Domains in MEPS study group

\begin{tabular}{|lllllllll|}
\hline & NO & RN & Dur & CCP & RF & TTP & SSS & WPI \\
MEPS & 400 & $0 \%$ & 1.22 & $0 \%$ & $18 \%$ & 15.2 & 9.62 & 15.32 \\
& & & \pm 0.86 & & & \pm 2.3 & \pm 2.11 & \pm 3.34 \\
\hline
\end{tabular}

RN: rheumatoid nodules, Dur. duration, CCP: anti-CCP, RF: rheumatoid factor, TTP: tender trigger points, SSS: symptom severity score, WPI: widespread pain index.

Table 4: Different radiological findings in MEPS study group 


\begin{tabular}{|llllllllll|}
\hline Group & NO & Sr & Odp & Bt & Rj & Cs & ch & Ts & WA \\
\hline MEPS & 400 & $88 \%$ & 12 & $30 \%$ & $30 \%$ & $45 \%$ & $20 \%$ & $95 \%$ & 35 \\
\hline
\end{tabular}

Sr: Subperiosteal resorption, Odp: Osteolysis of distal phalanges, Bt; Brown tumours, Rj: Rugger jersey appearance of vertebral column, Cs :Calcaneal spurs, ch:chondrocalcinosis, Ts: Tuft spur-like excrescences. WA: wrist arthritis.

\section{Figures}

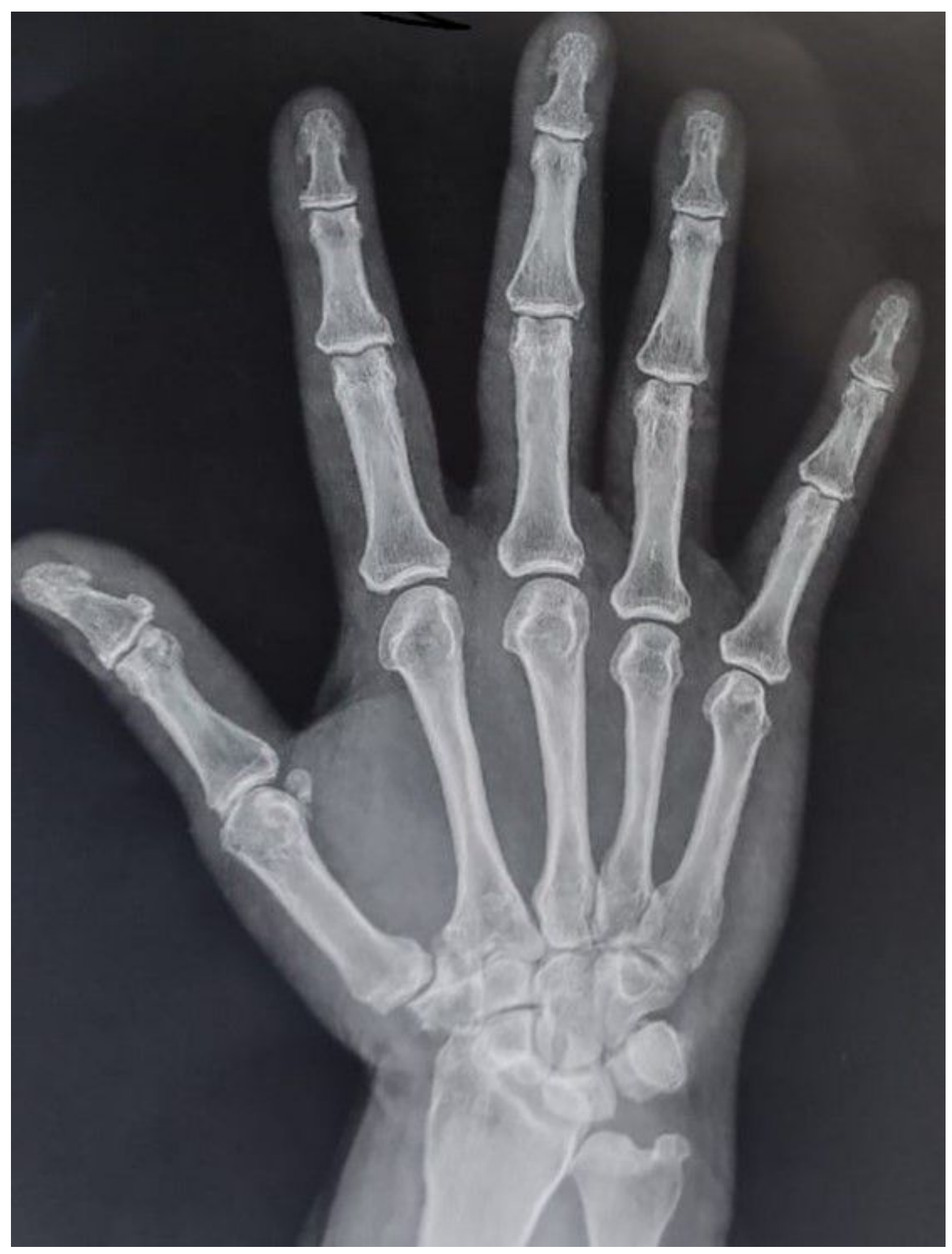




\section{Figure 1}

Mild subperiosteal bone resorption affecting radial aspects of the proximal and middle phalanges of the 2nd and 3rd fingers. No subligamentous resorption. Terminal tuft erosion (acro-osteolysis), changes in the carpus closely resembling those of rheumatoid arthritis, radiocarpal and scapho-trapezoid joint arthritis. as well as spur-like excrescences.

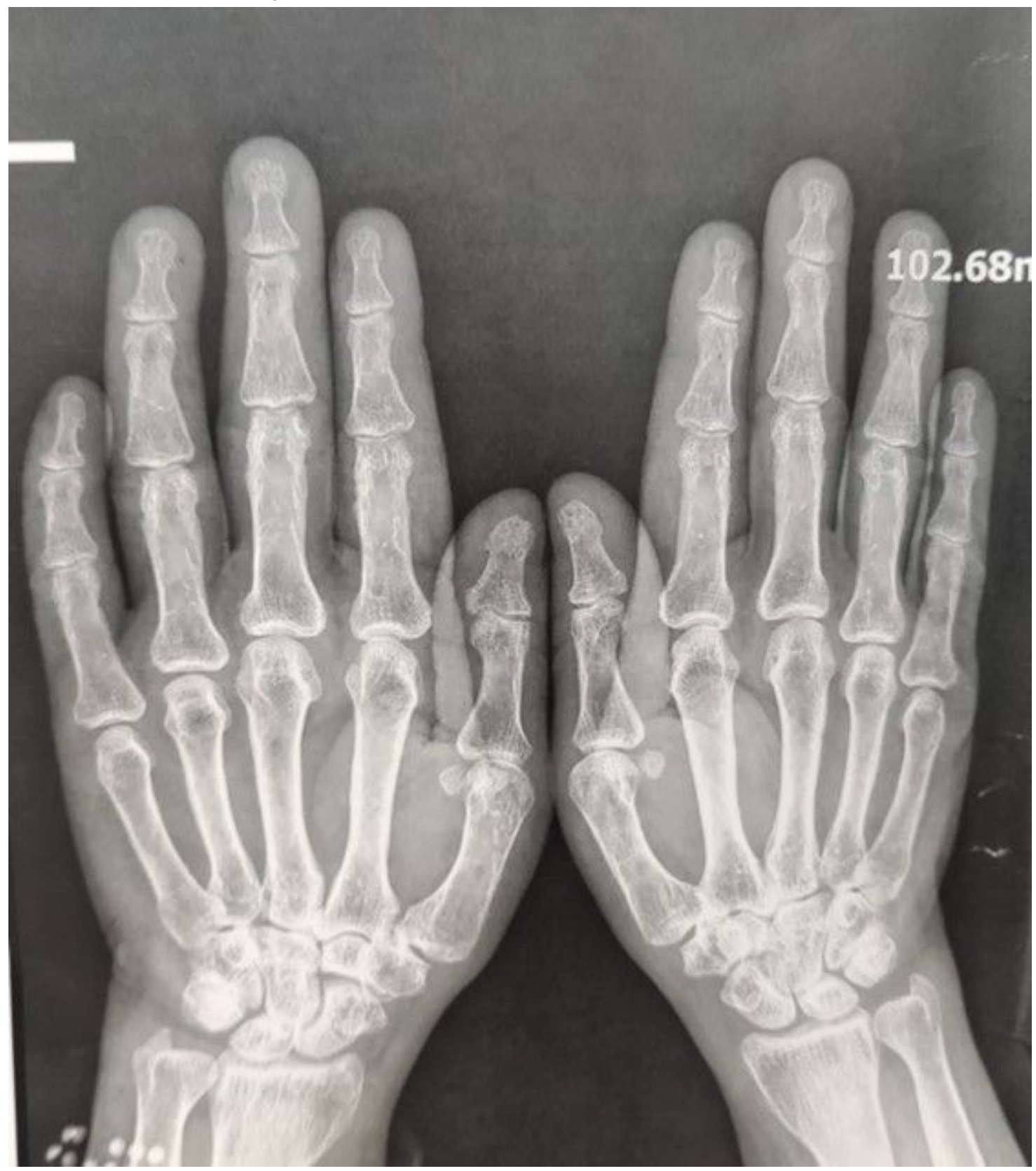

Figure 2

Subperiosteal bone resorption affecting radial aspects of the proximal and middle phalanges of the 2nd and 3rd fingers. Terminal tuft cysts (acro-osteolysis), as well as spur-like excrescences. 


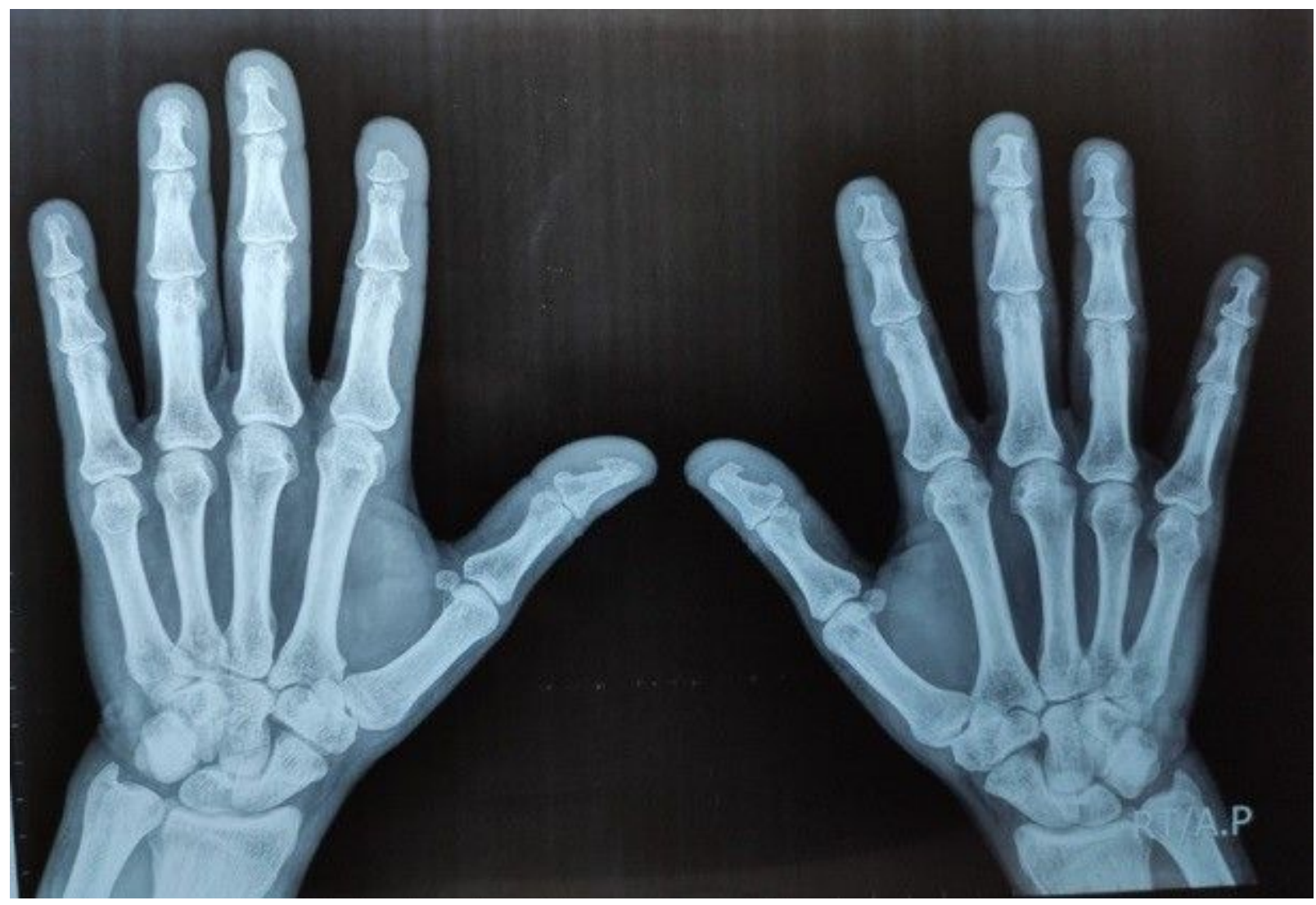

Figure 3

Acro osteolysis in index fingers, as well as spur-like excrescences bilaterally, with wrist arthritis.
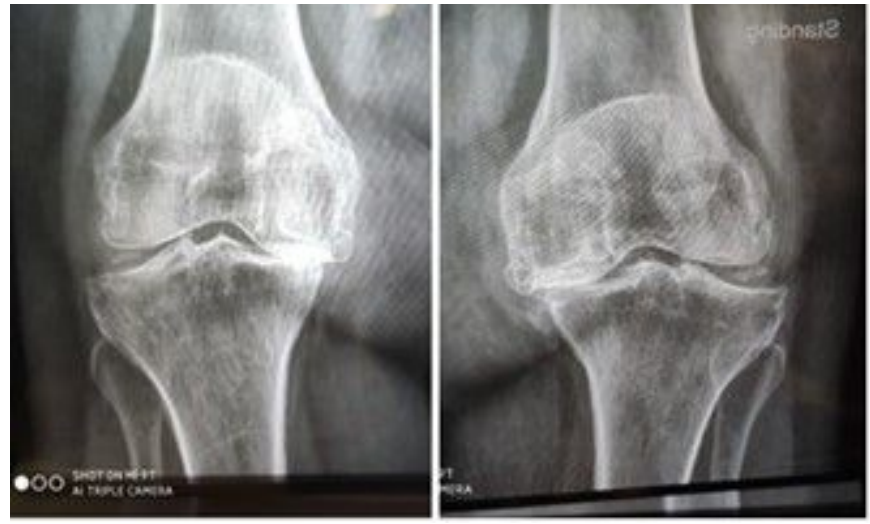

Figure 4

Plain $\mathrm{X}$ ray AP knee shows chondrocalcinosis in latral compartment of the knee. Intracortical resorption oval shape and narrow joint space.And bilateral posterior loose bodies. Subperiosteal bone resorption medial aspect femur 